\title{
The Impact of Gamification-Induced Users' Feelings on the Continued Use of mHealth Apps: A Structural Equation Model With the Self-Determination Theory Approach
}

Tong Wang ${ }^{1}$, BS; Lingye Fan², MD; Xu Zheng ${ }^{3}$, MSc; Wei Wang ${ }^{1}$, PhD; Jun Liang ${ }^{4}$, MS; Kai $\mathrm{An}^{3}, \mathrm{MSc}^{2} \mathrm{Mei} \mathrm{Ju}^{5}$, MS; Jianbo Lei ${ }^{6,7,8}, \mathrm{MD}, \mathrm{PhD}$

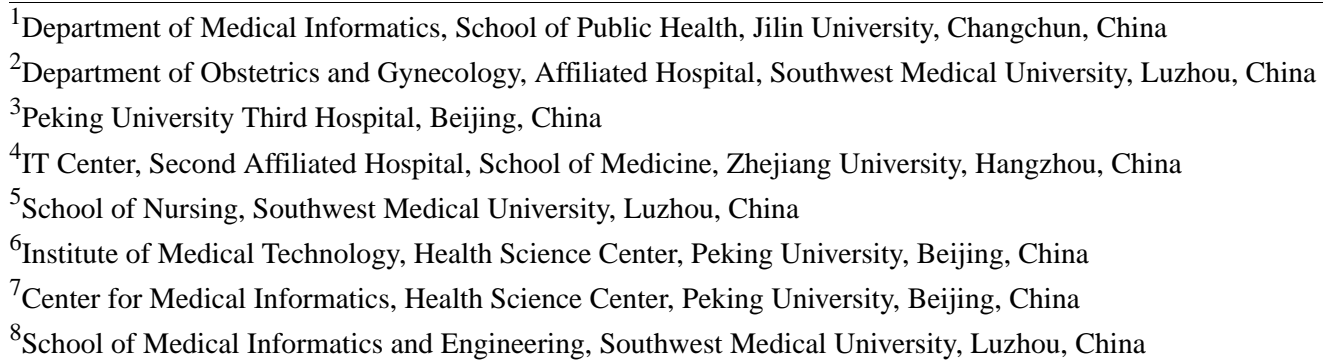

\section{Corresponding Author:}

Jianbo Lei, MD, PhD

Institute of Medical Technology, Health Science Center

Peking University

38 Xueyuan Rd, Haidian District

Beijing

China

Phone: 8682805901

Email: jblei@hsc.pku.edu.cn

\section{Abstract}

Background: Continued use of mHealth apps can achieve better effects in health management. Gamification is an important factor in promoting users' intention to continue using mHealth apps. Past research has rarely explored the factors underlying the continued use of mobile health (mHealth) apps and gamification's impact mechanism or path on continued use.

Objective: This study aimed to explore the factors influencing mHealth app users' intention to continue using mHealth apps and the impact mechanism and path of users' feelings induced by gamification on continued mHealth app use.

Methods: First, based on the expectation confirmation model of information system continuance, we built a theoretical model for continued use of mHealth apps based on users' feelings toward gamification. We used self-determination theory to analyze gamification's impact on user perceptions and set the resulting feelings (competence, autonomy, and relatedness) as constructs in the model. Second, we used the survey method to validate the research model, and we used partial least squares to analyze the data.

Results: A total of 2988 responses were collected from mHealth app users, and 307 responses were included in the structural equation model after passing the acceptance criteria. The intrinsic motivation for using mHealth apps is significantly affected by autonomy $(\beta=.312 ; P<.001)$, competence $(\beta=.346 ; P<.001)$, and relatedness $(\beta=.165 ; P=.004)$ induced by gamification. The intrinsic motivation for using mHealth apps has a significant impact on satisfaction $(\beta=.311, P<.001)$ and continuance intention $(\beta=.142 ; P=.045)$; furthermore, satisfaction impacts continuance intention significantly $(\beta=.415 ; P<.001)$. Confirmation has a significant impact on perceived usefulness $(\beta=.859 ; P<.001)$ and satisfaction $(\beta=.391 ; P<.001)$, and perceived usefulness has a significant impact on satisfaction $(\beta=.269 ; P<.001)$ and continuance intention $(\beta=.273 ; P=.001)$. The mediating effect analysis showed that in the impact path of the intrinsic motivation for using the mHealth apps on continuance intention, satisfaction plays a partial mediating role $(\beta=.129 ; P<.001)$, with a variance accounted for of 0.466 .

Conclusions: This study explored the impact path of users' feelings induced by gamification on the intention of continued mHealth app use. We confirmed that perceived usefulness, confirmation, and satisfaction in the classical continued use theory for nonmedical information systems positively affect continuance intention. We also found that the path and mechanism of users' 
feelings regarding autonomy, competence, and relatedness generated during interactions with different gamification elements promote the continued use of mHealth apps.

(J Med Internet Res 2021;23(8):e24546) doi: 10.2196/24546

\section{KEYWORDS}

mHealth app; continued use; continuance intention; gamification; self-determination theory (SDT); expectation confirmation model of information system continuance (ECM-ISC); PLS-SEM

\section{Introduction}

\section{Background}

Global health continues to face significant challenges, and health management is an important research direction. In 2016, 41 million people died of noncommunicable diseases globally, equivalent to $71 \%$ of global deaths [1]. One study showed that after 1 year of health management intervention, subjects' risks of hypertension and hyperglycemia were reduced by $42.78 \%$ and $31.13 \%$, respectively [2]. Moreover, every dollar invested in health management can reduce the cost of medical care by US \$1 to \$3 [3]. With mobile health (mHealth) technology maturity, the traditional health management model has gradually shifted to a management model based on mobile health.

With the popularization of smartphones, mHealth centered around mHealth apps has become an important means of health management. Related research is also increasing every year, mainly focusing on the health management effect, framework design, and user behavior regarding mHealth apps. According to estimates, more than 250,000 mHealth apps were on the market in 2016. It is estimated that there will be 4.68 billion mobile phone users worldwide in 2019, and the number of mHealth apps will achieve an annual growth rate of $41 \%$ between 2015 and 2020 [4]. mHealth can provide users with health intervention [5] and effective health evaluation indicators [6] and improve the communication between patients and health care professionals [7]. The number of studies on mHealth apps is also increasing every year. As of July 6, 2020, there were 688 articles related to mHealth apps in PubMed. Most studies have explored the intervention effect of mHealth apps [8-11]. Some studies focus on the development and design of mHealth apps to increase personalization and efficiency in health management services [12]. A few studies analyzed the acceptance and continued use of mHealth apps [13,14].

The effectiveness of health management based on an mHealth app is affected by user behavior. Short-term use can hardly achieve the expected goal of health management. However, the continued use of an mHealth app is not optimistic; most users only use it 4 times, and $25 \%$ use it only once after installation [14]. Therefore, it is essential to explore the influencing factors on users' continued use of mHealth apps for the sustainable development of mHealth. Previous studies have explored the factors influencing the continued use of different types of apps. The technology acceptance model, expectation confirmation theory, unified theory of acceptance and use of technology, and other theories have been used to explore the influencing factors of continued app use, such as mobile instant messaging [15], mobile social apps [16], mobile social tourism and shopping [17], and paid mobile apps [18]. Compared to other apps,
mHealth apps have different application scenarios and purposes, so it is unknown whether the research conclusions in other fields apply to mHealth apps. Therefore, some studies have explored the influencing factors of the continued mHealth app use from the aspects of quality, usability, user perception, and social impact. With the increasing number of new elements incorporated into applications, gamification is widely used in mHealth apps because it is believed to have the potential to promote continued use [19]. Out of 1000 mHealth apps, 772 contain at least 1 gamification element [20]. However, the effect of gamification in different apps is diverse [21], so whether gamification in mHealth apps has a positive impact on continued use needs to be explored.

Studies on the influence of gamification on mHealth apps' continued use are limited. Previous studies have shown that gamification has a significant impact on the intention to continue using other types of apps [20,22,23], but whether it has the same effect on mHealth apps needs to be verified. Therefore, studies are gradually trying to explain the impact of gamification on behavioral intention in mHealth apps. For example, based on motivational feedback, some studies analyzed the factors influencing the continued use of fitness apps-an important type of mHealth app in terms of effective, informational, and social feedback-and proved the effect of gamification on affective feedback [22]. Other studies analyzed the effects of utilitarian benefits, hedonic benefits, and social benefits of gamified fitness apps on users' continuance intention and expanded the analysis of multiple impacts of social benefits on users' psychology and cognition [24]. Although previous research has substantially increased the understanding of the relationship between gamification and continued use, there are some limitations. Gamification does not cause all individuals to have the same subjective feelings and encourage them to continue using mHealth apps [25]. There is less discussion about the impact of different types of gamification on users' diverse feelings, and the resulting explanation of the different effects of gamification is insufficient. Therefore, we propose the following research questions:

1. What are the impact factors on continuance intention for gamified mHealth apps? What is the relationship between the impact factors?

2. By what path does users' feeling induced by gamification affect users' continuance intention toward mHealth apps?

To answer these questions, we built a research model to explain how users' feelings induced by gamification affect the continuance intention for mHealth apps based on the expectation confirmation model of information system continuance (ECM-ISC) and self-determination theory (SDT). Specifically, SDT is used to analyze the impact of different types of 
gamification elements on users' feelings, and different feelings are set as constructs added to the model to explore the impact mechanism and path of gamification on the continued mHealth app use.

\section{Significance of This Study}

Based on the ECM-ISC model, we introduced SDT theory and innovatively used this composite model to explain psychological aspects of gamification elements that affect the motivation to use mHealth apps to promote the continuance intention of mHealth app use. Previous studies on continuance mainly focused on information systems such as hospital information systems and massive open online courses that focused on improving efficiency. There is insufficient research on the factors influencing the continued use induced by mHealth app gamification. Second, we linked gamification with the continued use model through SDT and added the users' feelings caused by different gamification elements into the research model. This paper proposes a model to explain the impact of users' feelings toward gamification on continued mHealth app use and explores the impact mechanism and path of gamification, an emerging information technology method combined with behavior change theory, on the continued mHealth app use.

We first conducted an empirical test on the continuance model of the gamified mHealth app and, for the first time, verified the applicability of the classic model ECM-ISC in the mHealth app field for Chinese users. Compared with other studies on continuance, we did not choose a specific app for verification and included more apps to increase the applicability of the results. Second, we analyzed the impact of users' feelings induced by gamification on promoting continued mHealth app use and provided a reference for mHealth app designers and researchers to promote users' continuance intention for the mHealth app and improve the effect of users' health management.

\section{Research Model and Hypotheses}

Based on the ECM-ISC, combined with SDT, we explored the impact factors of the continued use of the gamified mHealth app. The research model and assumptions are shown in Figure 1. F-1a aims to verify the applicability of ECM-ISC in the mHealth app, and F-1b explores the impact factors of feelings induced by gamification based on SDT.

Figure 1. Research model.

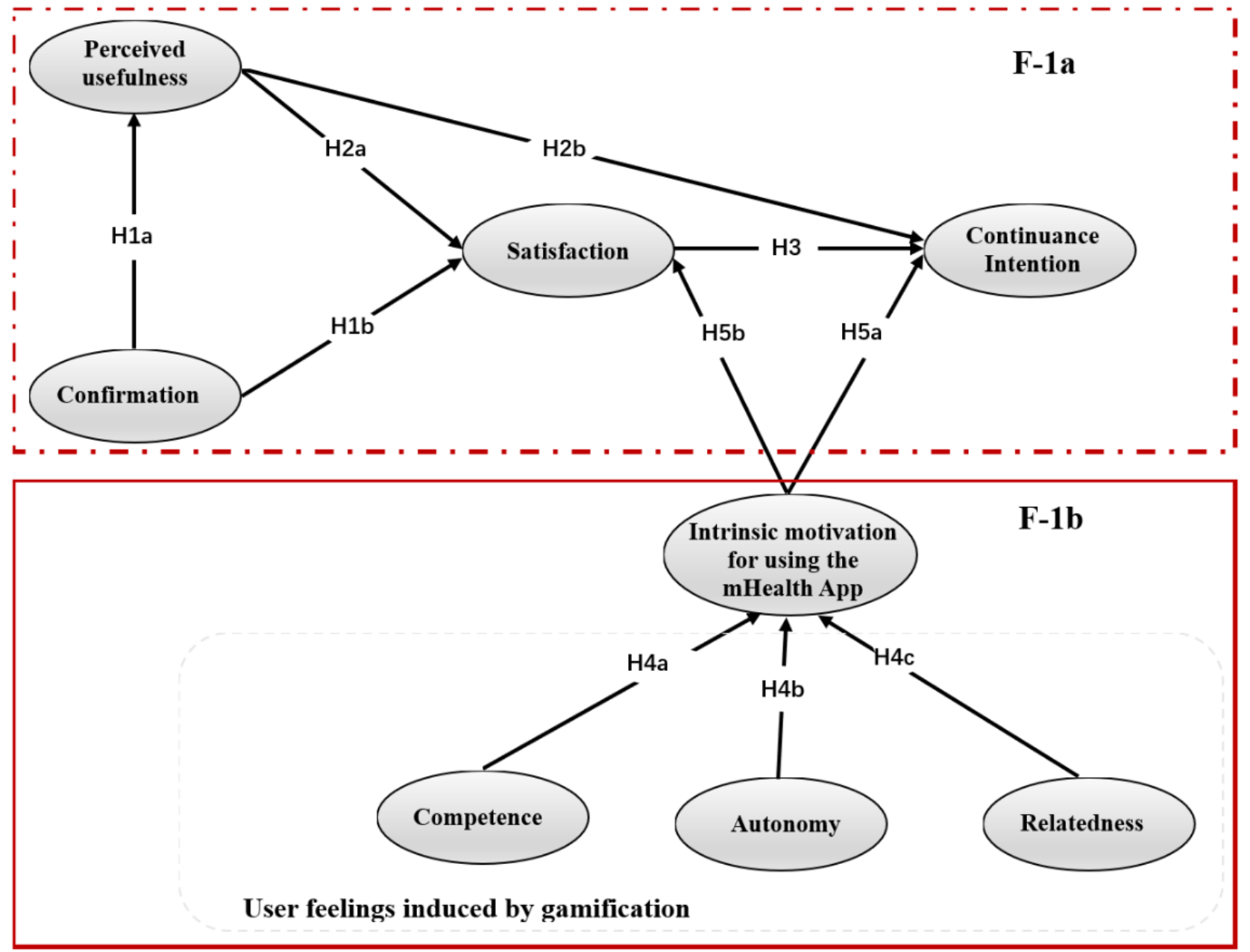




\section{Hypotheses in ECM-ISC}

The ECM-ISC (Figure 1, see F-1a) explains the process by which users generate continuance intention. Referring to the study by Bhattacherjee [26], the definitions of each construct are as follows: (1) confirmation is defined as users' perception of the congruence between expectations of mHealth apps use and its actual performance, (2) perceived usefulness refers to users' perception of the expected benefits of mHealth app use, (3) satisfaction is users' affect (feelings about) regarding prior mHealth apps use, (4) continuance intention is the user's willingness and tendency to continue using the mHealth app. Previous studies have revealed the relationship between these factors in nonmedical information systems [26]. Therefore, we believe that the same following assumptions exist in the mHealth app:

1. H1a: Confirmation positively influences perceived usefulness.

2. H1b: Confirmation positively influences satisfaction.

3. H2a: Perceived usefulness influences satisfaction.

4. H2b: Perceived usefulness influences continuance intention.

5. H3: Satisfaction influences continuance intention.

\section{Hypothesis of Constructs Generated by Gamification Elements}

SDT is a theory about human motivation and personality that highlights the importance of human-evolved inner resources for personality development and behavioral self-regulation. SDT defines 3 innate psychological needs: the needs for competence, autonomy, and relatedness [27]. SDT asserts that external things allow users to have autonomy, competence, and relatedness by satisfying innate psychological needs. The 3 feelings increase humans' intrinsic motivation [28]. Gamification is the application of game design elements in nongame contexts, so the essence of gamification in mHealth app is a design method of app. The gamification element is its manifestation, and it is objective within the app. The direct stimulus of behavior motivation is the user's personality and feelings rather than external factors. Therefore, we need to explore the effect of gamification on users' feelings and then learn its impacts on behavior motivation. To achieve this goal, we chose 5 of the most common gamification elements as research objects. According to the characteristics of the different elements, this paper analyzed the impact of gamification on users' feelings. In the context of this research, autonomy refers to gamification providing users with the right to choose and set specific gamification contents independently and to determine their health management content and set goals; therefore, users have autonomy over their behavior [28]. Health management is the primary goal of the mHealth app, so we mainly explore the user's right to choose and self-controlled behaviors in health management goals.

The common achievement or progression-oriented gamification elements include points, medals, and leaderboards (PML) [21]. Therefore, we assume that the user's competence will be affected after experiencing the above gamification elements. In the context of this article, competence means that users obtain gamified feedback after completing app health management tasks, and this feedback allows uses to understand their health management capabilities. Furthermore, social networking is a social-oriented gamification element, which can leverage the functions of friending, commenting, and sharing experiences [21]. In mHealth apps, the above functions are generally utilized by the health community. This process reduces a sense of isolation from others and satisfies user needs for relatedness, making users feel relatedness [28]. Furthermore, according to SDT, competence, autonomy, and relatedness can increase the intrinsic motivation to use the mHealth app, described as follows:

1. H4a: Competence induced by gamification positively influences intrinsic motivation for using the mHealth app.

2. H4b: Autonomy induced by gamification positively influences intrinsic motivation for using the mHealth app.

3. H4c: Relatedness induced by gamification positively influences intrinsic motivation for using the mHealth app.

\section{Relationship Between Intrinsic Motivation for Using the mHealth app and Satisfaction and Continuance Intention}

Intrinsic motivation drives human behaviors, emphasizing that humans carry out certain activities based on their personalities and feelings [27]. Feelings are not easy to change, so intrinsic motivation is persistent; therefore, the intrinsic motivations of the mHealth app promote continuance intention. Under the influence of their feelings and interests, users will actively use the mHealth app, resulting in satisfactory evaluations. Therefore, we hypothesize the following:

1. H5a: Intrinsic motivation for using the mHealth app positively influences continuance intention.

2. H5b: Intrinsic motivation for using the mHealth app positively influences satisfaction.

\section{Methods}

\section{Measurement Instrument}

This study constructed a new theoretical model to explore how users' feelings induced by gamification promote the continued use of mHealth apps. It was necessary to select appropriate observable variables for the constructs to verify our model. To develop the measurement instrument of the survey, we adapted previously validated scales to our research context. To design the measurement instrument reasonably, we adjusted the previously validated scales according to the experimental purpose. For the feelings caused by gamification elements, items for competence were drawn from McAuley et al [29]. Items for autonomy were sourced from several studies [29-31]. Items for relatedness were from 3 studies [30-32]. Items for intrinsic motivation for using mHealth apps were from Lin [33]. Scales for ECM-ISC, items for perceived usefulness, satisfaction, confirmation, and continuance intention were drawn from validated scales $[26,34,35]$. We used the 7-point Likert scale to measure the items with anchors ranging from 1 (strongly disagree) to 7 (strongly agree). We used the initial questionnaire to presurvey 17 testers and modified the scale based on evaluations and suggestions to form the final questionnaire (see Multimedia Appendix 1). We used the data collection service 
from China's largest online survey platform to manage the survey [36].

\section{Research Target and Data Collection}

The study subjects have used an mHealth app in the past 3 months. From January 20 to February 23, 2020, we used WeChat to conduct a snowball sampling survey, posting the link to the questionnaire on the WeChat moments and WeChat group. A total of 2988 questionnaires were returned, mainly distributed across 29 regions of China. In addition, 11 responses from Chinese users were also received from the United States, Australia, and Austria. We excluded responses that included the following characteristics: no mHealth app experience in the past 3 months $(n=1981,66.30 \%)$, repeat internet protocols (IP) $(n=18,0.60 \%)$, non-mHealth app $(n=85,2.84 \%)$, use of fewer than 3 gamification elements $(n=571,19.11 \%)$, and completion within 3 minutes $(n=26,0.87 \%)$. Finally, we obtained a valid questionnaire $(n=307,10.27 \%)$. No mHealth app experience means that users replied that they had not used the mHealth app in the past 3 months. A non-mHealth app means the app used by the user was not a mHealth app. We determined whether the app is an mHealth app according to the classification system of Apple's App Store. If the app used by the user was not included in the "health and fitness" and "medical" categories, it was regarded as a "non-mHealth app." The data inclusion and exclusion process are shown in Figure 2. In addition, although we selected users who have used at least 3 gamification elements, some users still needed to answer a few items that include a gamification element that users did not use. For the items involving in the gamification elements that participants did not use, we input point 4 (uncertainty) on the 7-point Likert scale. This study was approved by the Biomedical Ethics Committee of Peking University, and the subjects were aware of the study's purpose and process.

Figure 2. Data inclusion and exclusion process. IP: internet protocol; mHealth: mobile health.
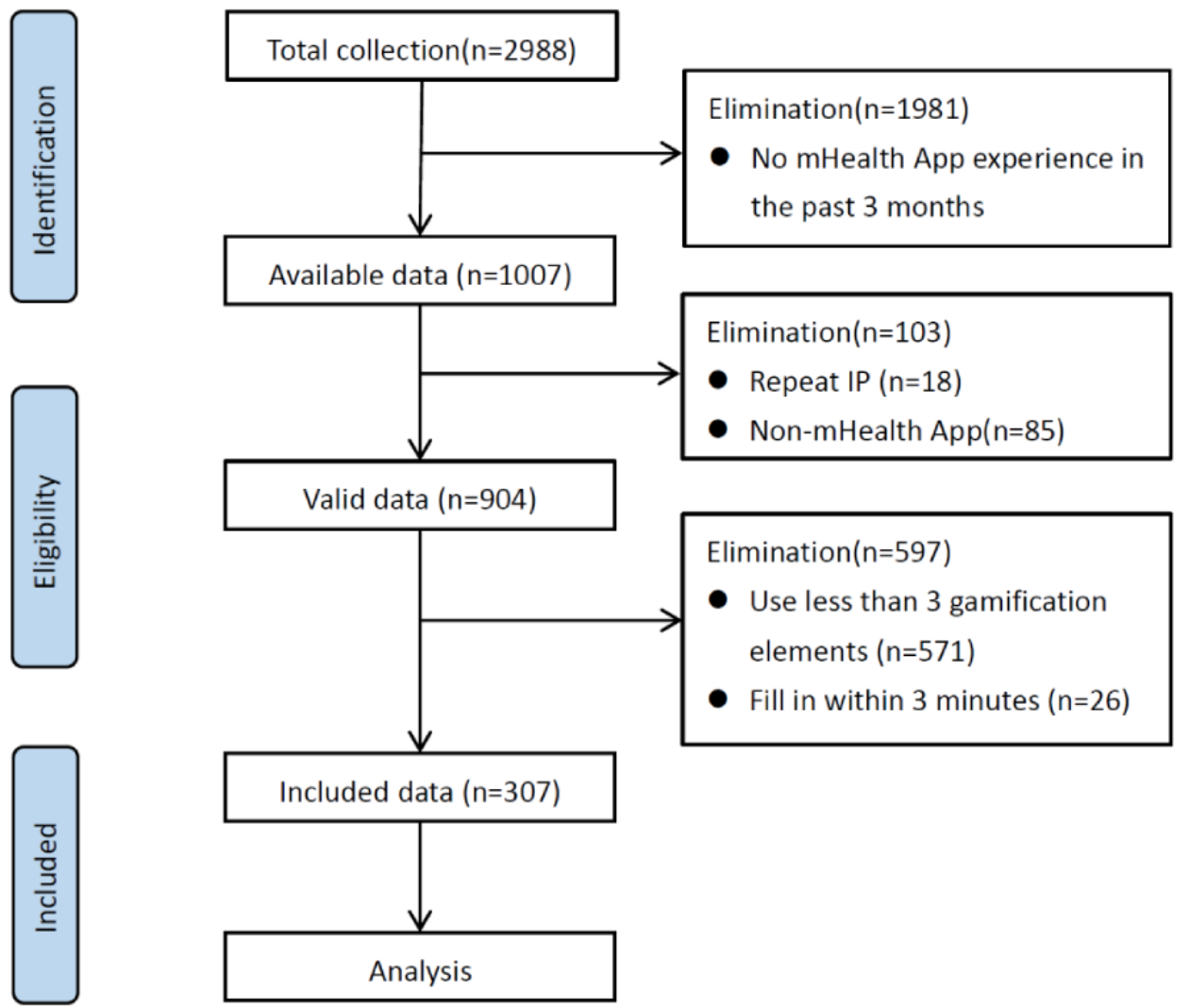

\section{Data Analysis}

The partial least squares structural equation model (PLS-SEM) is more suitable for exploratory research than the covariance-based structural equation model [37]. PLS-SEM can deal with non-normal data and complex models with more latent variables [38] and has lower sample requirements [10]. Our research is exploratory, and the data are not strictly normally distributed; therefore, this study uses PLS-SEM analysis with SmartPLS (version 3.2.8; SmartPLS GmbH).

\section{Results}

\section{Demographic Information}

The demographic information for the included samples is shown in Table 1 . We divided the apps into 4 categories according to their functions, including menstruation and pregnancy management, fitness and diet management, online consultation, etc. We also investigated the users' health status during follow-up to explore whether health status has a regulatory effect on continued use. The users' health was indirectly measured by perceived health using a 7-point Likert scale from 1 (very unhealthy) to 7 (very healthy), with an average of 5.35 (SD 1.193). 
Table 1. Demographic information.

\begin{tabular}{|c|c|}
\hline Characteristics & Users, $\mathrm{n}(\%)$ \\
\hline \multicolumn{2}{|l|}{ Gender } \\
\hline Male & $174(56.7)$ \\
\hline Female & $133(43.3$ \\
\hline \multicolumn{2}{|l|}{ Age } \\
\hline$\leq 30$ & $101(32.9)$ \\
\hline $31-45$ & $132(43.0)$ \\
\hline $46-60$ & $67(21.8)$ \\
\hline$\geq 61$ & $7(2.3)$ \\
\hline \multicolumn{2}{|l|}{ Education } \\
\hline High school & $4(1.3)$ \\
\hline Junior college & $22(7.2)$ \\
\hline College & $130(42.3)$ \\
\hline Master's degree and above & $151(49.2)$ \\
\hline \multicolumn{2}{|l|}{ Income } \\
\hline$\leq 2500$ & $60(19.5)$ \\
\hline $2501-5000$ & $28(9.1)$ \\
\hline $5001-8000$ & $57(18.6)$ \\
\hline $8001-30,000$ & $125(40.7)$ \\
\hline$\geq 30,001$ & $37(12.1)$ \\
\hline \multicolumn{2}{|l|}{ Frequency } \\
\hline$\leq 1$ time per week & $83(27.0)$ \\
\hline 2-3 times per week & 95 (30.9) \\
\hline 4-5 times per week & $58(18.9)$ \\
\hline 6-7 times per week & $45(14.7)$ \\
\hline$>7$ times per week & $26(8.5)$ \\
\hline \multicolumn{2}{|l|}{ Occupation } \\
\hline Students & $58(18.9)$ \\
\hline Medicine-related personnel & $70(22.8)$ \\
\hline Public servants and clerks & $77(25.1)$ \\
\hline Commercial and service personnel & $28(9.1)$ \\
\hline Professional technical personnel & $66(21.5)$ \\
\hline Manual workers & $4(1.3)$ \\
\hline Unemployed and other personnel & $4(1.3)$ \\
\hline \multicolumn{2}{|l|}{ Type of mHealth app } \\
\hline Menstruation and pregnancy management & $11(3.6)$ \\
\hline Fitness and diet management & $212(69.1)$ \\
\hline Online consultation & $37(12.1)$ \\
\hline Others & $47(15.3)$ \\
\hline
\end{tabular}




\section{Analysis Result of Hypothesized Model}

\section{Measurement Model}

The reliability and validity of the questionnaire were measured by confirmatory factor analysis. The results are shown in Table 2. To test the model's reliability, we calculated Cronbach's alpha and composite reliability based on the score of each item. A value of 0.5 represents acceptable reliability, and 0.7 represents good reliability [39]. To verify the convergent validity, we calculated the project load and the value of average extracted variance (AVE). The larger the factor loading, the greater the influence of the observed variable on the latent variable. The commonly adopted acceptance standard is 0.7 . AVE represents the degree of aggregation of different items in the same construct, and a value greater than 0.5 represents acceptable convergence validity [39]. As shown in Table 2, all the values of Cronbach's alpha and composite reliabilities are above 0.8 , the AVE of each construct is above 0.6, and the loading weights for each item are above 0.7 , indicating good reliability for all constructs and good convergent validity. As shown in Table 3, we compare the correlation between the AVE square root of each construct and other constructs to show that the observed variable of the same construct is not related to other latent variables. The results show that the square root of AVE of each structure is greater than the interconstruct correlations, showing good discriminant validity [40]. Therefore, we conclude that the results of the measurement model are sufficient to test the hypotheses in the model.

Considering that all constructs are measured by a scale, common method bias needs to be tested to eliminate systematic errors caused by the same data source and measurement environment. We performed a statistical analysis that is suitable for the PLS-SEM model. We added a common factor to the model, which contains all items, and calculated each indicator's substantive variances explained by the principal construct and by the method [41]. The average substantive variance of the indicators is 0.904 , while the average method-based variance is 0.007 . The ratio of substantive variance to method variance is approximately 129:1 (see Multimedia Appendix 2). Given the small magnitude and insignificance of method variance, we believe that there is no serious common method bias in this study. The multicollinearity was checked by calculating the variance inflation factor (VIF) [42]. The VIF values of all the regressions were all substantially below the cutoff value of 10 , which means there are no severe multicollinearity problems (see Multimedia Appendix 2). 
Table 2. Construct reliability and convergent validity.

\begin{tabular}{|c|c|c|c|c|}
\hline Construct and items & Factor loadings & Composite reliability & Average variance extracted & Cronbach alphas \\
\hline \multicolumn{5}{|l|}{ Autonomy (AUT) } \\
\hline AUT1 & 0.908 & 0.935 & 0.827 & 0.896 \\
\hline AUT2 & 0.914 & & & \\
\hline AUT3 & 0.906 & & & \\
\hline \multicolumn{5}{|c|}{ Competence (COMP) } \\
\hline COMP1 & 0.806 & 0.891 & 0.6732 & 0.817 \\
\hline COMP2 & 0.874 & & & \\
\hline COMP3 & 0.883 & & & \\
\hline \multicolumn{5}{|l|}{ Relatedness (REL) } \\
\hline REL1 & 0.847 & 0.936 & 0.786 & 0.909 \\
\hline REL2 & 0.864 & & & \\
\hline REL3 & 0.925 & & & \\
\hline REL4 & 0.907 & & & \\
\hline \multicolumn{5}{|c|}{ Continuance intention (CI) } \\
\hline CI1 & 0.888 & 0.925 & 0.754 & 0.891 \\
\hline $\mathrm{CI} 2$ & 0.799 & & & \\
\hline $\mathrm{CI} 3$ & 0.884 & & & \\
\hline CI4 & 0.900 & & & \\
\hline \multicolumn{5}{|c|}{ Confirmation (CONF) } \\
\hline CONF1 & 0.943 & 0.967 & 0.906 & 0.948 \\
\hline CONF2 & 0.968 & & & \\
\hline CONF3 & 0.945 & & & \\
\hline \multicolumn{5}{|c|}{ Intrinsic motivation for using the mHealth app (MOT) } \\
\hline MOT1 & 0.914 & 0.949 & 0.823 & 0.928 \\
\hline MOT2 & 0.926 & & & \\
\hline МОТ3 & 0.926 & & & \\
\hline MOT4 & 0.862 & & & \\
\hline \multicolumn{5}{|c|}{ Perceived usefulness (USE) } \\
\hline USE1 & 0.931 & 0.961 & 0.892 & 0.940 \\
\hline USE2 & 0.961 & & & \\
\hline USE3 & 0.941 & & & \\
\hline \multicolumn{5}{|l|}{ Satisfaction (SAT) } \\
\hline SAT1 & 0.932 & 0.949 & 0.861 & 0.919 \\
\hline SAT2 & 0.916 & & & \\
\hline SAT3 & 0.937 & & & \\
\hline
\end{tabular}


Table 3. Discriminant validity.

\begin{tabular}{|c|c|c|c|c|c|c|c|c|}
\hline & $\mathrm{AUT}^{\mathrm{a}}$ & $\mathrm{COMP}^{\mathrm{b}}$ & $\mathrm{CONF}^{\mathrm{c}}$ & $\mathrm{CI}^{\mathrm{d}}$ & $\mathrm{MOT}^{\mathrm{e}}$ & $U_{S E}^{f}$ & $\mathrm{REL}^{\mathrm{g}}$ & SAT $^{\mathrm{h}}$ \\
\hline AUT & $0.909^{\mathrm{i}}$ & & & & & & & \\
\hline COMP & 0.355 & 0.855 & & & & & & \\
\hline CONF & 0.445 & 0.490 & 0.952 & & & & & \\
\hline CI & 0.427 & 0.458 & 0.779 & 0.869 & & & & \\
\hline MOT & 0.464 & 0.546 & 0.760 & 0.710 & 0.907 & & & \\
\hline USE & 0.505 & 0.545 & 0.859 & 0.754 & 0.785 & 0.945 & & \\
\hline REL & 0.182 & 0.547 & 0.352 & 0.310 & 0.411 & 0.362 & 0.886 & \\
\hline SAT & 0.512 & 0.507 & 0.859 & 0.781 & 0.820 & 0.849 & 0.373 & 0.928 \\
\hline
\end{tabular}

aUT: autonomy.

${ }^{\mathrm{b}} \mathrm{COMP}$ : competence.

${ }^{\mathrm{c}} \mathrm{CONF}$ : confirmation.

${ }^{\mathrm{d}} \mathrm{CI}$ : continuance intention.

${ }^{\mathrm{e}}$ MOT: intrinsic motivation for using the mHealth app.

${ }^{\mathrm{f}} \mathrm{USE}$ : perceived usefulness.

${ }^{\mathrm{g}} \mathrm{REL}$ : relatedness.

$\mathrm{h}_{\text {SAT: satisfaction. }}$

${ }^{\mathrm{i}}$ Italics refers to the square roots of average variance extracted.

\section{Structural Model}

Through the bootstrapping analysis in PLS-SEM [43], we tested the hypotheses in the research model. As shown in Figure 3, there is an explanation for variance $\mathrm{R}^{2}$ and standardized path coefficient $\beta . R^{2}$ refers to the variance of endogenous latent variables that can be explained by exogenous latent variables. The exogenous latent variable explains the variance, and $\beta$ represents the correlation between the variables. First, regarding the applicability of ECM-ISC in an mHealth app, confirmation significantly affected both perceived usefulness and satisfaction, and perceived usefulness significantly affected satisfaction and continuance intention for the mHealth app. In addition,

satisfaction significantly impacted the continuance intention for the mHealth app. Therefore, H1a, H1b, H2a, H2b, and H3 were supported. The above results tested the applicability of ECM-ISC in the mHealth app. Second, the constructs related to gamification, competence, autonomy, and relatedness significantly influenced the intrinsic motivation for using an mHealth app. Therefore, $\mathrm{H} 4 \mathrm{a}, \mathrm{H} 4 \mathrm{~b}$, and $\mathrm{H} 4 \mathrm{c}$ were all supported. Intrinsic motivation for using an mHealth app significantly impacted satisfaction and continuance intention for the mHealth app; thus, H5a and H5b were supported. As shown in Figure 3, age, gender, education, income, occupation, and other social demographic characteristics have no significant moderating effects on the results. 
Figure 3. Analysis results of structural model. mHealth: mobile health.

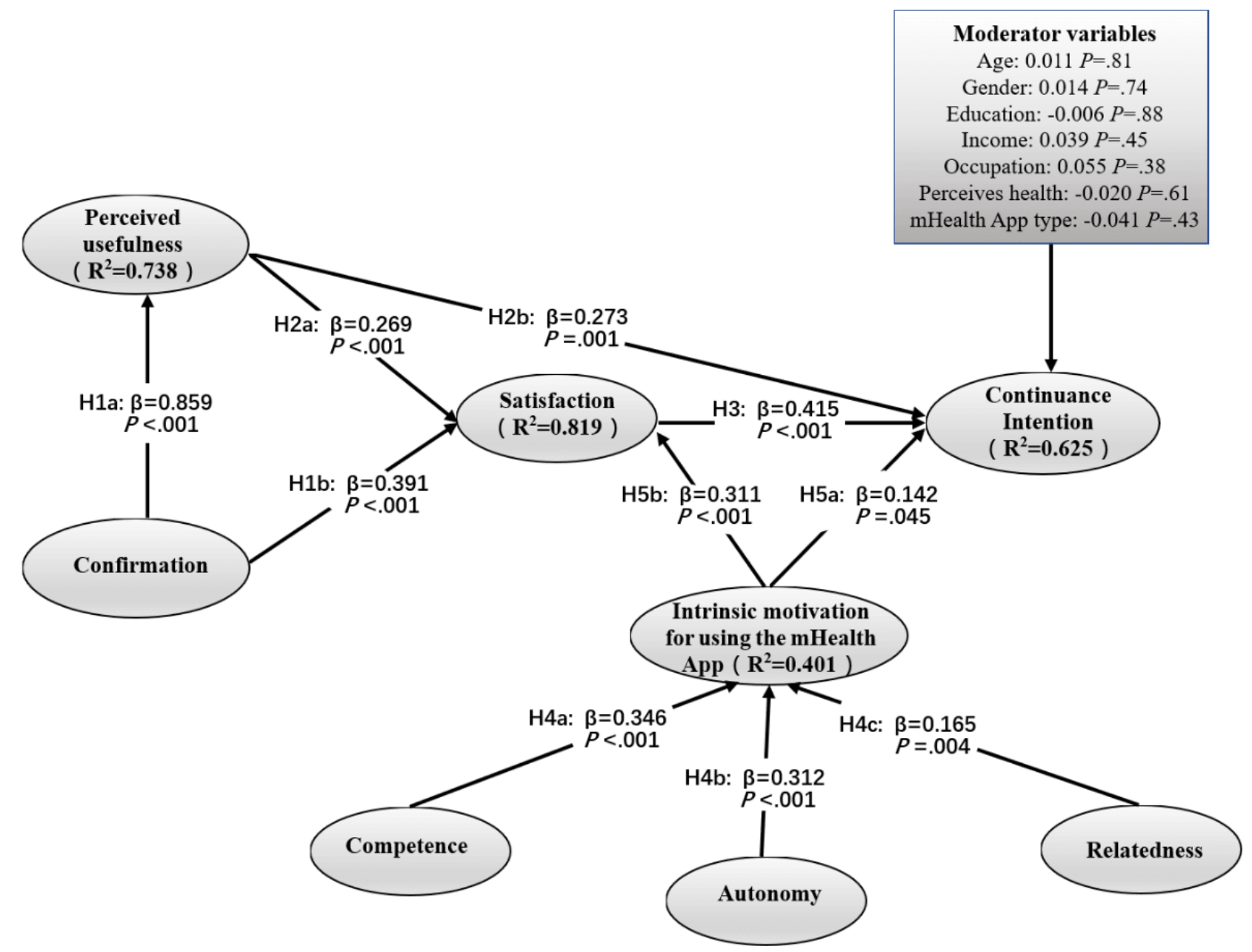

\section{Results of Mediation Analysis}

PLS-SEM and bootstrapping analysis results included direct effects, indirect effects, and total effects between variables. These allowed us to directly use variance accounted for (VAF) to analyze the mediating effects between variables. VAF measures the ratio of indirect effects to total effects. A VAF greater than 0.8 is generally considered a complete mediation effect, and a partial mediation effect is between 0.2 and 0.8 [44]. As shown in Table 4, all the effects were significant, whereas satisfaction was a partial mediator variable between motivation for using an mHealth app and continuance intention for the mHealth app and between perceived usefulness and continuance intention for the mHealth app. In addition, perceived usefulness was a partial mediator variable between confirmation and satisfaction.

Table 4. Mediation analysis.

\begin{tabular}{|c|c|c|c|c|c|c|c|}
\hline \multirow[t]{2}{*}{ Independent variable } & \multirow[t]{2}{*}{ Mediating variable } & \multirow[t]{2}{*}{ Dependent variable } & \multicolumn{2}{|c|}{ Indirect effects } & \multicolumn{2}{|c|}{ Total effects } & \multirow{2}{*}{ VAF $^{\mathrm{a}}$} \\
\hline & & & $\beta$ & $P$ value & $\beta$ & $P$ value & \\
\hline Intrinsic motivation & Satisfaction & Continuance & .129 & $<.001$ & .277 & $<.001$ & 0.466 \\
\hline Usefulness & Satisfaction & Continuance & .112 & .002 & .386 & $<.001$ & 0.290 \\
\hline Confirmation & Usefulness & Satisfaction & 231 & $<.001$ & .621 & $<.001$ & 0.372 \\
\hline
\end{tabular}

${ }^{\mathrm{a}}$ VAR: variance accounted for.

\section{Discussion}

\section{Most Current Gamification in the mHealth App Is Unattractive to Users}

In the study, 1007 users had mHealth app experience in the past 3 months, but 248 (24.63\%) users believed they had not used gamification elements. Auditing the questionnaires of the 248 users, we found that 109 (43.95\%) of them used apps that contained at least 1 gamification element. Gamification has not attracted the users' attention and interest because some gamification advocates believe that the golden combination of PML can work in any app [25], leading some designers to simply combine gamification elements applied in the mHealth 
app with no characteristics and without considering the users' unique needs and intrinsic motivations. Although gamification elements are widely used in mHealth apps, the design process lacks an understanding of gamification and users' feelings; therefore, they cannot attract users.

\section{The Impact Path of Gamification on Continuance Intention for mHealth Apps}

This study shows that users' autonomy, competence, and relatedness interacting with different gamification elements positively promote their motivation for using an mHealth app, followed by the continuance intention for the mHealth app. This path also explains the ineffectiveness of gamification on some users. For example, if users do not feel autonomy, competence, and relatedness in the process of interacting with gamification, then gamification will not have a significant impact on the continued use of an mHealth app for this type of user.

The explanation of the impact path is as follows. First, users hope to make personalized settings in the mHealth app, such as health management tasks and user image [45]. The personalized settings allow users to experience tailor-made services, and they cater to users' interests. Autonomy is generated during the process of experiencing gamification elements such as optional goals, which increases intrinsic motivation for using the mHealth app. Second, social interaction within the mHealth app promotes the users' intention for use, indicating that the more online friends and the more frequent the interactions between users, the more significant the impact of social interaction on users' mHealth app use $[46,47]$. Users share health management experiences through online communities. In experiencing gamification elements with social orientation, there are interactive behaviors among users. These behaviors help users receive support and care and feel relatedness, increasing the intrinsic motivation for using the mHealth app. Finally, this study confirmed that competence caused by gamification promoted motivation for using the mHealth app. The representative gamification elements that affect competence in this study are PML. In mHealth apps, users need to complete health management tasks (eg, exercise, diet control, etc) to obtain such elements. These elements show users' progress in health management, enhancing their competence and increasing their motivation to use mHealth apps. However, it is difficult for users to change their health status after completing a health management task, and long-term and frequent use can achieve the desired effect [14]. Therefore, it is very important to reasonably design gamification elements to induce users' sense of competence, relatedness, and autonomy, increasing users' motivation to use mHealth apps.

\section{The Influence Mechanism of Gamification on the Continuance Intention of the mHealth App}

In the continued use model of the gamified mHealth app, significant impacts include confirmation, perceived usefulness, satisfaction, motivation for using the mHealth app, autonomy, competence, relatedness, and continuance intention. Among them, satisfaction and motivation for using the mHealth app have direct effects on continuance intention. Satisfaction mediates the influence of motivation for using the mHealth app on continuance intention.

High intrinsic motivation means that users' interests and feelings, which are not easily affected by external factors, are satisfied. Therefore, intrinsic motivation increases satisfaction. Furthermore, it is more likely that the user's inner emotional motivation will continue to exist, so the intrinsic motivation for using the mHealth app promotes continuance intention. This is consistent with conclusions by Cruz et al [48] and and Chang [49]. The results of the mediating effect analysis showed that satisfaction is the mediating factor explaining continuance intention, which is consistent with the conclusions from Hsiao et al [16]. Therefore, we concluded that the motivation for using an mHealth app has both direct and indirect effects on mHealth app continuance intention.

\section{Limitations and Future Research}

First, we collected data using snowball sampling, and the questionnaire was distributed through WeChat. Although the participants were distributed across the country, the sample was not enough to represent all mHealth app users, which may cause bias in the results.

Second, although we explored the impact of 5 common gamification elements on users' feelings, we did not analyze the specific role of each element and the differences between the impact of each gamification element on users' feelings. However, research in this direction is of great significance for understanding gamification. Therefore, our subsequent research focus will distinguish the effects of different types of gamification elements on users' feelings and continuance intention.

\section{Conclusion}

Based on ECM-ISC and SDT, we established a theoretical model of gamified mHealth app continuance and validated the proposed research model. Specifically, this study not only verifies the applicability of ECM-ISC to mHealth apps but also confirms that perceived usefulness, confirmation, and satisfaction positively impact mHealth app continuance intention. In terms of the impact mechanism, the competence users experience in their interaction with achievement and progress-oriented gamification elements in mHealth application, the relatedness users experience during their interaction with social-oriented gamification elements, and the autonomy users experience in their interaction with personalized service gamification elements enhance the intrinsic motivation of using mHealth apps, thus promoting the continuance intention.

\section{Acknowledgments}

This study was sponsored by the National Natural Science Foundation of China (grants 81771937 and 81871455) and PKU-Baidu Fund (grants 2019BD008). It was partially supported by the Luzhou Science and Technology Bureau project, constructing a 
health big data platform and service system for the elderly care institutions integrated with medical care, and the Interdisciplinary Research Funding Program for Doctoral Students of Jilin University (101832020DJX081).

\section{Authors' Contributions}

TW performed the experiments and analyzed the data. TW, LF, and XZ contributed reagents, materials, or analysis tools and prepared the figures and tables. WW and J Lei conceived and designed the experiments. J Lei authored or reviewed drafts of the paper. J Liang, KA, and MJ made revisions to the manuscript. All authors approved the final version of the manuscript.

\section{Conflicts of Interest}

None declared.

\section{Multimedia Appendix 1}

Measurement instrument.

[DOCX File, 24 KB-Multimedia Appendix 1]

\section{Multimedia Appendix 2}

CMV and Multicollinearity analysis.

[DOCX File, 20 KB-Multimedia Appendix 2]

\section{Multimedia Appendix 3}

Total collection's demographic information.

[DOCX File, 17 KB-Multimedia Appendix 3]

\section{References}

1. World health statistics 2020: monitoring health for the SDGs, sustainable development goals. World Health Organization. 2020. URL: https://apps.who.int/iris/bitstream/handle/10665/332070/9789240005105-eng.pdf [accessed 2020-07-08]

2. Loeppke R, Edington DW, Bég S. Impact of the prevention plan on employee health risk reduction. Popul Health Manag 2010 Oct;13(5):275-284 [FREE Full text] [doi: 10.1089/pop.2010.0027] [Medline: 20879909]

3. Study: Wellness Programs Saved $\$ 1$ to $\$ 3$ per Dollar Spent. URL: https://www.shrm.org/resourcesandtools/hr-topics/ benefits/pages/wellness-dollars-saved.aspx [accessed 2020-07-08]

4. Liew MS, Zhang J, See J, Ong YL. Usability Challenges for Health and Wellness Mobile Apps: Mixed-Methods Study Among mHealth Experts and Consumers. JMIR Mhealth Uhealth 2019 Jan 30;7(1):e12160 [FREE Full text] [doi: 10.2196/12160] [Medline: 30698528]

5. Kao C, Liebovitz DM. Consumer Mobile Health Apps: Current State, Barriers, and Future Directions. PM R 2017 May;9(5S):S106-S115. [doi: 10.1016/j.pmrj.2017.02.018] [Medline: 28527495]

6. Xie J, Wen D, Liang L, Jia Y, Gao L, Lei J. Evaluating the Validity of Current Mainstream Wearable Devices in Fitness Tracking Under Various Physical Activities: Comparative Study. JMIR Mhealth Uhealth 2018 Apr 12;6(4):e94 [FREE Full text] [doi: 10.2196/mhealth.9754] [Medline: 29650506]

7. Marcolino MS, Oliveira JAQ, D'Agostino M, Ribeiro AL, Alkmim MBM, Novillo-Ortiz D. The Impact of mHealth Interventions: Systematic Review of Systematic Reviews. JMIR Mhealth Uhealth 2018 Jan 17;6(1):e23 [FREE Full text] [doi: 10.2196/mhealth.8873] [Medline: 29343463]

8. Buis LR, Dawood K, Kadri R, Dawood R, Richardson CR, Djuric Z, et al. Improving Blood Pressure Among African Americans With Hypertension Using a Mobile Health Approach (the MI-BP App): Protocol for a Randomized Controlled Trial. JMIR Res Protoc 2019 Jan 25;8(1):e12601 [FREE Full text] [doi: 10.2196/12601] [Medline: $\underline{30681965]}$

9. Kosse RC, Bouvy ML, Belitser SV, de Vries TW, van der Wal PS, Koster ES. Effective Engagement of Adolescent Asthma Patients With Mobile Health-Supporting Medication Adherence. JMIR Mhealth Uhealth 2019 Mar 27;7(3):e12411 [FREE Full text] [doi: 10.2196/12411] [Medline: 30916664]

10. Doocy S, Paik KE, Lyles E, Hei TH, Fahed Z, Winkler E, et al. Guidelines and mHealth to Improve Quality of Hypertension and Type 2 Diabetes Care for Vulnerable Populations in Lebanon: Longitudinal Cohort Study. JMIR Mhealth Uhealth 2017 Oct 18;5(10):e158 [FREE Full text] [doi: 10.2196/mhealth.7745] [Medline: 29046266]

11. Jiang Y, West BT, Barton DL, Harris MR. Acceptance and Use of eHealth/mHealth Applications for Self-Management Among Cancer Survivors. Stud Health Technol Inform 2017;245:131-135 [FREE Full text] [Medline: 29295067]

12. Klee P, Bussien C, Castellsague M, Combescure C, Dirlewanger M, Girardin C, et al. An Intervention by a Patient-Designed Do-It-Yourself Mobile Device App Reduces HbA1c in Children and Adolescents with Type 1 Diabetes: A Randomized Double-Crossover Study. Diabetes Technol Ther 2018 Dec;20(12):797-805. [doi: 10.1089/dia.2018.0255] [Medline: $\underline{30403495}$ 
13. Morano JP, Clauson K, Zhou Z, Escobar-Viera CG, Lieb S, Chen IK, et al. Attitudes, Beliefs, and Willingness Toward the Use of mHealth Tools for Medication Adherence in the Florida mHealth Adherence Project for People Living With HIV (FL-mAPP): Pilot Questionnaire Study. JMIR Mhealth Uhealth 2019 Jul 03;7(7):e12900 [FREE Full text] [doi: 10.2196/12900] [Medline: 31271150]

14. Vaghefi I, Tulu B. The Continued Use of Mobile Health Apps: Insights From a Longitudinal Study. JMIR Mhealth Uhealth 2019 Aug 29;7(8):e12983 [FREE Full text] [doi: 10.2196/12983] [Medline: 31469081]

15. Oghuma AP, Libaque-Saenz CF, Wong SF, Chang Y. An expectation-confirmation model of continuance intention to use mobile instant messaging. Telematics and Informatics 2016 Feb;33(1):34-47. [doi: 10.1016/j.tele.2015.05.006]

16. Hsiao CH, Chang JJ, Tang KY. Exploring the influential factors in continuance usage of mobile social Apps: Satisfaction, habit, and customer value perspectives. Telematics and Informatics 2016 May;33(2):342-355. [doi: 10.1016/j.tele.2015.08.014]

17. Hew JJ, Leong LY, Tan GWH, Lee VH, Ooi KB. Mobile social tourism shopping: A dual-stage analysis of a multi-mediation model. Tourism Management 2018 Jun;66:121-139. [doi: 10.1016/j.tourman.2017.10.005]

18. Hsu CL, Lin JCC. What drives purchase intention for paid mobile apps? - An expectation confirmation model with perceived value. Electronic Commerce Research and Applications 2015 Jan;14(1):46-57. [doi: 10.1016/j.elerap.2014.11.003]

19. Ibarra-Herrera CC, Carrizosa A, Yunes-Rojas JA, Mata-Gómez MA. Design of an app based on gamification and storytelling as a tool for biology courses. Int J Interact Des Manuf 2019 Jun 13;13(4):1271-1282. [doi: 10.1007/s12008-019-00600-8]

20. Zhang Y, Xiao S, Zhou G. User continuance of a green behavior mobile application in China: An empirical study of Ant Forest. Journal of Cleaner Production 2020 Jan;242:118497. [doi: 10.1016/j.jclepro.2019.118497]

21. Koivisto J, Hamari J. The rise of motivational information systems: A review of gamification research. International Journal of Information Management 2019 Apr;45:191-210. [doi: 10.1016/j.ijinfomgt.2018.10.013]

22. Hassan L, Dias A, Hamari J. How motivational feedback increases user's benefits and continued use: A study on gamification, quantified-self and social networking. International Journal of Information Management 2019 Jun;46:151-162. [doi: 10.1016/j.ijinfomgt.2018.12.004]

23. Lee C, Lee K, Lee D. Mobile Healthcare Applications and Gamification for Sustained Health Maintenance. Sustainability 2017 May 08;9(5):772. [doi: 10.3390/su9050772]

24. Hamari J, Koivisto J. Why do people use gamification services? International Journal of Information Management 2015 Aug;35(4):419-431. [doi: 10.1016/j.ijinfomgt.2015.04.006]

25. Cugelman B. Gamification: what it is and why it matters to digital health behavior change developers. JMIR Serious Games 2013;1(1):e3 [FREE Full text] [doi: 10.2196/games.3139] [Medline: 25658754]

26. Bhattacherjee A. Understanding Information Systems Continuance: An Expectation-Confirmation Model. MIS Quarterly 2001 Sep;25(3):351. [doi: 10.2307/3250921]

27. Ryan RM, Deci EL. Self-determination theory and the facilitation of intrinsic motivation, social development, and well-being. American Psychologist 2000;55(1):68-78. [doi: 10.1037/0003-066X.55.1.68]

28. Ryan, Deci. Intrinsic and Extrinsic Motivations: Classic Definitions and New Directions. Contemp Educ Psychol 2000 Jan;25(1):54-67. [doi: 10.1006/ceps.1999.1020] [Medline: 10620381]

29. McAuley E, Duncan T, Tammen VV. Psychometric properties of the Intrinsic Motivation Inventory in a competitive sport setting: a confirmatory factor analysis. Res Q Exerc Sport 1989 Mar;60(1):48-58. [doi: 10.1080/02701367.1989.10607413] [Medline: 2489825]

30. Inchamnan W. An Analysis of Creative Process Learning in Computer Game Activities Through Player Experiences. IAFOR J Educ 2016 Sep;4(2):119-139. [doi: 10.22492/ije.4.2.07]

31. La Guardia JG, Ryan RM, Couchman CE, Deci EL. Within-person variation in security of attachment: A self-determination theory perspective on attachment, need fulfillment, and well-being. Journal of Personality and Social Psychology 2000;79(3):367-384. [doi: 10.1037/0022-3514.79.3.367]

32. Lee Y, Lee J, Hwang Y. Relating motivation to information and communication technology acceptance: Self-determination theory perspective. Computers in Human Behavior 2015 Oct;51:418-428. [doi: 10.1016/j.chb.2015.05.021]

33. Lin CP, Tsai YH, Chiu CK. Modeling Customer Loyalty from an Integrative Perspective of Self-Determination Theory and Expectation-Confirmation Theory. J Bus Psychol 2009 May 1;24(3):315-326. [doi: 10.1007/s10869-009-9110-8]

34. Rahi S, Abd. Ghani M. Integration of expectation confirmation theory and self-determination theory in internet banking continuance intention. JSTPM 2019 Oct 2;10(3):533-550. [doi: 10.1108/jstpm-06-2018-0057]

35. Hsiao JL, Chen RF. Understanding Determinants of Health Care Professionals' Perspectives on Mobile Health Continuance and Performance. JMIR Med Inform 2019 Mar 18;7(1):e12350 [FREE Full text] [doi: 10.2196/12350] [Medline: 30882353]

36. Questionnaire star_Questionnaire/onlien examination. URL: https://www.wjx.cn/ [accessed 2021-07-28]

37. Wu T, Deng Z, Zhang D, Buchanan PR, Zha D, Wang R. Seeking and using intention of health information from doctors in social media: The effect of doctor-consumer interaction. Int J Med Inform 2018 Jul;115:106-113. [doi: 10.1016/j.ijmedinf.2018.04.009] [Medline: 29779712]

38. Hair JF, Ringle CM, Sarstedt M. PLS-SEM: Indeed a Silver Bullet. The Journal of Marketing Theory and Practice 2011 Mar 15;19(2):139-152. [doi: 10.2753/MTP1069-6679190202] 
39. Wu T, Deng Z, Chen Z, Zhang D, Wu X, Wang R. Predictors of Patients' Loyalty Toward Doctors on Web-Based Health Communities: Cross-Sectional Study. J Med Internet Res 2019 Sep 03;21(9):e14484 [FREE Full text] [doi: 10.2196/14484] [Medline: 31482855$]$

40. Fornell C, Larcker DF. Evaluating Structural Equation Models with Unobservable Variables and Measurement Error. Journal of Marketing Research 2018 Nov 28;18(1):39-50. [doi: 10.1177/002224378101800104]

41. Liang, Saraf, Hu, Xue. Assimilation of Enterprise Systems: The Effect of Institutional Pressures and the Mediating Role of Top Management. MIS Quarterly 2007;31(1):59. [doi: 10.2307/25148781]

42. Fang J, Zhao Z, Wen C, Wang R. Design and performance attributes driving mobile travel application engagement. International Journal of Information Management 2017 Aug;37(4):269-283. [doi: 10.1016/j.ijinfomgt.2017.03.003]

43. Wu T, Deng Z, Chen Z, Zhang D, Wang R, Wu X. Predictors of Patients' Intention to Interact With Doctors in Web-Based Health Communities in China: Cross-Sectional Study. J Med Internet Res 2019 Jun 07;21(6):e13693 [FREE Full text] [doi: 10.2196/13693] [Medline: 31199296$]$

44. Hair JF, Hult GTM, Ringle CM, Sarstedt M. A primer on partial least squares structural equation modeling (PLS-SEM), 2nd edition. Los Angeles: SAGE Publications; 2017.

45. Milward J, Deluca P, Drummond C, Watson R, Dunne J, Kimergård A. Usability Testing of the BRANCH Smartphone App Designed to Reduce Harmful Drinking in Young Adults. JMIR Mhealth Uhealth 2017 Aug 08;5(8):e109 [FREE Full text] [doi: 10.2196/mhealth.7836] [Medline: 28790022]

46. Mendiola MF, Kalnicki M, Lindenauer S. Valuable features in mobile health apps for patients and consumers: content analysis of apps and user ratings. JMIR Mhealth Uhealth 2015 May 13;3(2):e40 [FREE Full text] [doi: 10.2196/mhealth.4283] [Medline: 25972309]

47. Hamari J, Koivisto J. "Working out for likes": An empirical study on social influence in exercise gamification. Computers in Human Behavior 2015 Sep;50:333-347. [doi: 10.1016/j.chb.2015.04.018]

48. Cruz P, Salo J, Gallego PM, Laukkanen T. Heavy users of e-banking and Customer Experience Management: evidences on intrinsic motivation. IJEB 2010;8(2):187. [doi: 10.1504/ijeb.2010.032093]

49. Chang CC, Liang CY, Yan CF, Tseng JS. The Impact of College Students' Intrinsic and Extrinsic Motivation on Continuance Intention to Use English Mobile Learning Systems. Asia-Pacific Edu Res 2013 May;22(2):181-192. [doi: 10.1007/s40299-012-0011-7]

\author{
Abbreviations \\ AVE: average extracted variance \\ ECM-ISC: expectation confirmation model of information system continuance \\ IP: internet protocol \\ mHealth: mobile health \\ PLS-SEM: partial least squares structural equation model \\ PML: points, medals, and leaderboards \\ SDT: self-determination theory \\ VAF: variance accounted for \\ VIF: variance inflation factor
}

Edited by R Kukafka; submitted 24.09.20; peer-reviewed by M Schmidt-Kraepelin, T Wu; comments to author 17.10.20; revised version
received 09.12.20; accepted 13.07.21; published 12.08 .21
Please cite as:
Wang T, Fan L, Zheng X, Wang W, Liang J, An K, Ju M, Lei J
The Impact of Gamification-Induced Users' Feelings on the Continued Use of mHealth Apps: A Structural Equation Model With the
Self-Determination Theory Approach
J Med Internet Res 2021;23(8):e24546
URL: $\underline{\text { https://www.jmir.org/2021/8/e24546 }}$
doi: $\underline{10.2196 / 24546}$
PMID:

(CTong Wang, Lingye Fan, Xu Zheng, Wei Wang, Jun Liang, Kai An, Mei Ju, Jianbo Lei. Originally published in the Journal of Medical Internet Research (https://www.jmir.org), 12.08.2021. This is an open-access article distributed under the terms of the Creative Commons Attribution License (https://creativecommons.org/licenses/by/4.0/), which permits unrestricted use, distribution, and reproduction in any medium, provided the original work, first published in the Journal of Medical Internet Research, is 
properly cited. The complete bibliographic information, a link to the original publication on https://www.jmir.org/, as well as this copyright and license information must be included. 